\title{
Baker's yeast $\beta$-glucan supplementation increases monocytes and cytokines post-exercise: implications for infection risk?
}

\author{
K. C. Carpenter, W. L. Breslin, T. Davidson, A. Adams and B. K. McFarlin* \\ Laboratory of Integrated Physiology, University of Houston, 3855 Holman Street, Houston, TX 77204, USA
}

(Submitted 4 January 2012 - Final revision received 24 February 2012 - Accepted 6 March 2012 - First published online 10 May 2012)

\begin{abstract}
Strenuous aerobic exercise is known to weaken the immune system, and while many nutritional supplements have been proposed to boost post-exercise immunity, few are known to be effective. The purpose of the present study was to evaluate whether $10 \mathrm{~d}$ of supplementation with a defined source of baker's yeast $\beta$-glucan (BG, Wellmune WGP ${ }^{\circledR}$ ) could minimise post-exercise immunosuppression. Recreationally active men and women ( $n$ 60) completed two $10 \mathrm{~d}$ trial conditions using a cross-over design with a $7 \mathrm{~d}$ washout period: placebo (rice flour) and baker's yeast BG $(250 \mathrm{mg} / \mathrm{d}$ of $\beta-1,3 / 1,6$-glucans derived from Saccharomyces cerevisiae) before a bout of cycling ( $49 \pm 6 \mathrm{~min})$ in a hot $\left(38 \pm 2^{\circ} \mathrm{C}\right.$ ), humid $(45 \pm 2 \%$ relative humidity) environment. Blood was collected at baseline (before supplement), pre- (PRE), post(POST) and $2 \mathrm{~h}(2 \mathrm{H})$ post-exercise. Total and subset monocyte concentration was measured by four-colour flow cytometry. Plasma cytokine levels and lipopolysaccharide (LPS)-stimulated cytokine production were measured using separate multiplex assays. Total (CD14 ${ }^{+}$) and pro-inflammatory monocyte concentrations $\left(\mathrm{CD} 14^{+} / \mathrm{CD} 16^{+}\right)$were significantly greater at POST and $2 \mathrm{H}(P<0 \cdot 05)$ with BG supplementation. BG supplementation boosted LPS-stimulated production of IL-2, IL-4, IL-5 and interferon- $\gamma$ (IFN- $\gamma$ ) at PRE and POST $(P<0 \cdot 05)$. Plasma IL-4, IL-5 and IFN- $\gamma$ concentrations were greater at $2 \mathrm{H}$ following BG supplementation. It appears that $10 \mathrm{~d}$ of supplementation with BG increased the potential of blood leucocytes for the production of IL-2, IL-4, IL- 5 and IFN- $\gamma$. The key findings of the present study demonstrate that BG may have potential to alter immunity following a strenuous exercise session.
\end{abstract}

Key words: $\beta$-Glucan supplementation: Immunity: Wellmune: Infection susceptibility

Strenuous aerobic exercise or a physically exhaustive work session is known to disrupt innate immune function for up to $24 \mathrm{~h}^{(1,2)}$. Environmental extremes exacerbate the typical exercise response and may further weaken the immune system ${ }^{(3)}$. Reduction in mucosal immunity, in addition to altered innate immune cells, results in an 'open window ${ }^{\text {(4) }}$ where an individual may be more susceptible to infections. Both natural killer cells and monocytes contribute to the innate immune response and are altered during recovery from exercise ${ }^{(2,5,6)}$. Becoming ill after exercise can result in lost training days, reductions in performance and lost working days. In the case of physical labourers, police officers, firefighters and soldiers, such sickness may increase the risk of job-related injuries and fatalities due to fatigue. Over the past decade, a number of laboratories including our own have explored the use of many different dietary supplements in mitigating post-exercise immune suppression ${ }^{(7-9)}$. $\beta$-Glucans (BG) have repeatedly been (tested) for immune-enhancing/modulatory properties ${ }^{(10-14)}$. However, there is great variation in these studies with regard to the efficacy of BG to modulate immunity ${ }^{(15)}$.
A likely reason for this variation is the many sources (and therefore types) of BG tested. BG are carbohydrates composed of glucose molecules linked together by several different types of chemical linkages resulting in either a linear or branched structure. For example, BG from cereal grains (oats and barley) have a linear structure, while BG from fungal sources (mushrooms and yeast) have a 1,3/1,6 linkage pattern with varying degrees of side-chain branches attached to the backbone. The frequency and length of side-chain branches have been shown to have important implications for biological activity; in general, the higher the degree of branching, the more biologically active the $\mathrm{BG}^{(11)}$. The baker's yeast-derived BG used in the present study is a commercially available preparation that has been structurally characterised in detail and is known to have a high degree of side-chain branching ${ }^{(15)}$.

Previous studies using baker's yeast BG have reported that supplementation after running a marathon reduces the number of symptomatic upper respiratory tract infection days compared with a placebo (PL) over a 4 -week period ${ }^{(16)}$. Similar

Abbreviations: $2 \mathrm{H}, 2 \mathrm{~h}$ post-exercise time point; baseline, time point before supplementation; BG, $\beta$-glucan; GM-CSF, granulocyte, monocyte colonystimulating factors; HR, heart rate; IFN- $\boldsymbol{\gamma}$, interferon- $\boldsymbol{\gamma}$; LPS, lipopolysaccharide; PBMC, peripheral blood mononuclear cells; POST, immediately postexercise time point; PRE, after supplementation and before exercise time point; $T_{\mathrm{C}}$, core body temperature; $\mathrm{VO}_{2 \text { peak }}$, maximal aerobic fitness level. 
reductions in upper respiratory tract infection symptoms have been noted in forest firefighters who were supplementing their diet with $\mathrm{BG}^{(17)}$. The purpose of the present study was to determine whether $10 \mathrm{~d}$ of supplementation with a particulate form of baker's yeast BG (Wellmune WGP) improved the innate immune system response to a strenuous bout of exercise in a hot, humid environment. In order to evaluate the innate immune system, we focused on monocytes, lipopolysaccharide (LPS)-stimulated cytokine production by whole-blood culture, and plasma cytokine concentrations.

\section{Materials and methods \\ Approach to the problem}

The present study used a double-blind, cross-over design to evaluate the ability of baker's yeast BG to counter post-exercise disruptions in innate immunity. Key outcome measures were changes in monocyte concentration, LPS-stimulated wholeblood cytokine production and plasma cytokine concentration. Each subject completed both conditions (BG or PL) using a $10 \mathrm{~d}$, pre-exercise supplementation period and a $7 \mathrm{~d}$ washout period between trials. A given subject completed their two experimental exercise trials at the same time of day, following an overnight fast $(>8 \mathrm{~h})$ and abstention from exercise $(>24 \mathrm{~h})$. Upon arrival at the laboratory, subjects were asked to complete a $24 \mathrm{~h}$ food recall to compare dietary intake. We did not find any significant condition differences for total energy intake or any of the macronutrients. We opted to have the subjects exercise in a hot, humid environment in order to increase physiological stress associated with exercise in a manner that matched our subject population. For the present study, we elected to use 'recreationally active' college students who were regularly exposed to stressful situations that may weaken the immune system over time.

\section{Determination of sample size}

The present study was conducted according to the guidelines laid down in the Declaration of Helsinki and all procedures involving human subjects/patients were approved by the University of Houston Committee for the Protection of Human Subjects. Written informed consent was obtained from all subjects/patients. Before completing the present study, we performed a pilot study using twelve men and women to evaluate which immune variables (natural killer cells, T-cells, monocytes, cytokines, etc.) were most likely to change with baker's yeast BG (Wellmune WGP) supplementation before exercise (BK McFarlin, unpublished data). From this testing, we found interesting trends for monocytes, but little change for natural killer and T-cells. Of the monocyte responses, the variable with the smallest effect size was monocyte concentration (0.35). Using this effect size and a statistical power of $0 \cdot 80$, we found that we would need a minimum of fifty-five subjects to detect significant differences between the BG and PL conditions. We increased the sample size of the present study to sixty-nine subjects in order to account for any poten- tial dropouts, while adding additional statistical power. Over the course of the study, nine subjects were either excused for failing to follow study protocols or asked to discontinue participation in the study (Fig. 1).

\section{Subjects}

In the present study, sixty male and female individuals who were recreationally active completed all testing. Fig. 1 demonstrates the recruitment and selection process of subjects in the present study. Subject demographics are presented in Table 1. After expressing interest in the study, subjects reported to the laboratory for a baseline testing session to measure their height, weight and body composition, and determine their maximal aerobic fitness level $\left(\mathrm{VO}_{2 \text { peak }}\right)$. Body composition was determined via a whole-body dual-energy X-ray absorptiometry scan (Hologic Discovery W). $\mathrm{VO}_{2 \text { peak }}$ was determined using a graded exercise test on an electronically braked cycle ergometer (Velotron) using a standard graded protocol from our laboratory ${ }^{(2,8,18,19)}$. When a subject's $\mathrm{VO}_{2 \text { peak }}$ was classified as 'average' or 'good' for their age/sex group (according to the standards of the American College of Sports Medicine $)^{(20)}$, the subject was approved for further participation in the study. Individuals who had 'excellent' fitness for their age group were excluded from further participation.

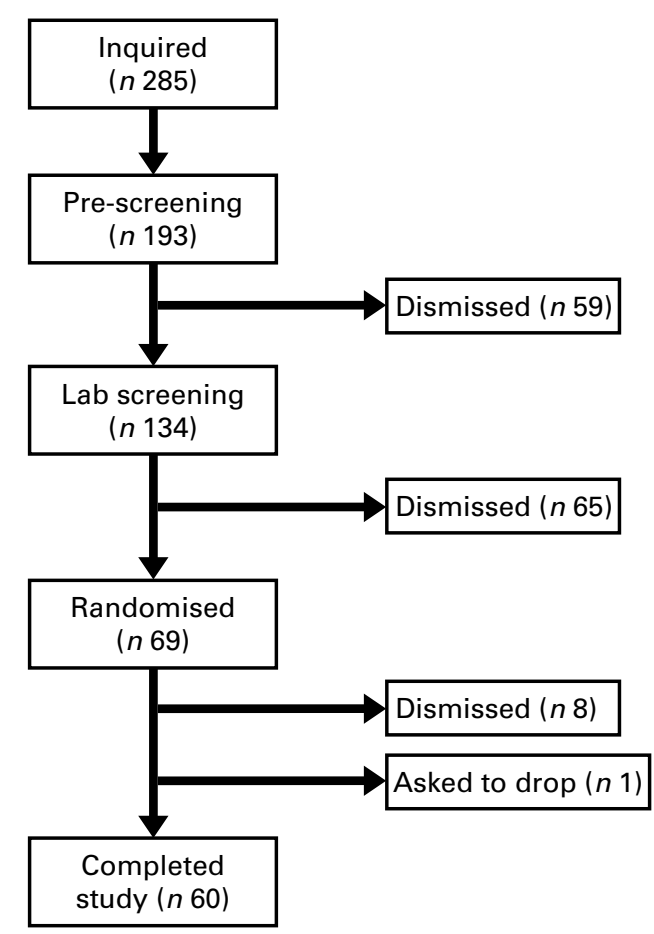

Fig. 1. Consort diagram. This is the consort recruitment diagram for the present study. The subject pool for the study started with 285 interested individuals and was reduced to the final number of sixty based on inclusion/exclusion criteria and subject attrition. Subjects completed two $10 \mathrm{~d}$ supplementation periods with either baker's yeast $\beta$-glucan $(250 \mathrm{mg} / \mathrm{d})$ or placebo (rice flour). After each supplementation period, subjects completed an experimental exercise trial in a hot, humid environment. Blood samples were collected and analysed for markers of immune system function. 
Table 1. Subject characteristics

(Mean values and standard deviations)

\begin{tabular}{|c|c|c|c|c|}
\hline \multirow[b]{2}{*}{ Characteristics } & \multicolumn{2}{|c|}{ Men ( $n$ 29) } & \multicolumn{2}{|c|}{ Women ( $n$ 31) } \\
\hline & Mean & SD & Mean & SD \\
\hline \% Minority* & \multicolumn{2}{|c|}{$55 \%$ (16 of 29$)$} & \multicolumn{2}{|c|}{$32 \%(10$ of 31$)$} \\
\hline Age (years) & 23 & 5 & 22 & 4 \\
\hline Height $(\mathrm{cm})$ & $176 \cdot 2$ & $7 \cdot 3$ & $165 \cdot 2$ & $5 \cdot 9$ \\
\hline Body mass (kg) & $75 \cdot 7$ & $8 \cdot 1$ & $63 \cdot 1$ & $7 \cdot 2$ \\
\hline BMI $\left(\mathrm{kg} / \mathrm{m}^{2}\right)$ & $24 \cdot 4$ & $1 \cdot 7$ & 22.5 & 1.9 \\
\hline $\mathrm{VO}_{2 \max }(\mathrm{ml} / \mathrm{kg}$ per min) & $45 \cdot 7$ & $5 \cdot 1$ & $42 \cdot 4$ & $6 \cdot 1$ \\
\hline Heart rate max (bpm) & 179 & 14 & 184 & 13 \\
\hline Resistance max (W) & 258 & 45 & 204 & 43 \\
\hline Fat mass $(\mathrm{kg})$ & $9 \cdot 4$ & 3.9 & $14 \cdot 0$ & 3.4 \\
\hline Lean mass $(\mathrm{kg})$ & $57 \cdot 2$ & $6 \cdot 6$ & $42 \cdot 7$ & $5 \cdot 3$ \\
\hline Bone mineral content $(\mathrm{kg})$ & $2 \cdot 4$ & 0.4 & $1 \cdot 8$ & 0.2 \\
\hline$\%$ Body fat & $14 \cdot 2$ & 4.7 & $23 \cdot 7$ & 4.0 \\
\hline
\end{tabular}

max, Maximal value observed during the graded exercise test; bpm, beats per min. ${ }^{*} \%$ Minority represents the percentage of the subject population that was of selfreported minority status.

\section{Baker's yeast $\beta$-glucan supplement}

A commercially available, particulate form of baker's yeast BG was used in the present study. BG was prepared by Biothera (the Immune Health Company) and consisted of $\beta-1,3 / 1,6$-glucans derived from baker's yeast (Saccharomyces cerevisiae) ${ }^{(15)}$. Rice flour was used as a PL because it was similar in colour and appearance to the BG supplement. Both supplements were in powder form and were packed into VegeCap ${ }^{\circledR}$ capsules before packaging in individual coded bottles. Biothera randomly assigned a number to each condition before shipping to the University of Houston. Double-blind protocols were maintained until all study data had been collected in raw form and submitted to Biothera. Upon receipt of the raw data report, Biothera unblinded the University of Houston study staff so that they could interpret the findings of the study based on condition.

In addition to blinding of the study staff, trial conditions were completed in a random, counterbalanced order to minimise possible trial-order effects. Each subject completed two conditions: $10 \mathrm{~d}$ of PL $(250 \mathrm{mg} / \mathrm{d}$ of rice flour $)$ and $10 \mathrm{~d}$ of baker's yeast BG (250 mg/d of Wellmune WGP). Supplement conditions were separated by a $7 \mathrm{~d}$ washout period. Subjects were instructed to consume the supplement with water, not within $2 \mathrm{~h}$ of consuming a meal and at generally the same time each day. Subjects were provided a form to document their daily supplement intake.

\section{Experimental exercise trial}

Subjects completed a $10 \mathrm{~d}$ supplementation period (PL or BG) before each exercise trial. Exercise consisted of up to $60 \mathrm{~min}$ of cycling in a hot $\left(38 \pm 2{ }^{\circ} \mathrm{C}\right)$, humid ( $45 \pm 2 \%$ ) environment. During the exercise trial, physiological stress was monitored by measurement of the core body temperature (rectal, $T_{\mathrm{C}}$ ), the heart rate (HR) and the rating of perceived exertion ${ }^{(19)}$. Rectal $T_{\mathrm{C}}$ was measured using a disposable thermistor and HR was measured by telemetry (Polar). Exercise intensity was set to fixed wattage that elicited approximately $65 \%$ of $\mathrm{VO}_{2 \text { peak }}$ based on the HR response. Exercise sessions were stopped if the subject reached a $T_{\mathrm{C}}>39 \cdot 2^{\circ} \mathrm{C}$. Subjects were allowed ad libitum intake of water during the exercise trials. Hydration status was monitored before and after each exercise trial by measuring $\mathrm{Hb}$ and haematocrit using a portable analyser (Stanbio's Hemopoint H2; Stanbio Laboratory). In the event that the subject stopped before $60 \mathrm{~min}$, their exercise duration was matched for their next exercise trial. For example, if a subject reached their $T_{\mathrm{C}}$ cut-off at $48 \mathrm{~min}$ for trial 1 , then in trial 2 , they would be asked to stop at $48 \mathrm{~min}$. This was designed to ensure that similar amounts of exercise stress were applied between the trials.

\section{Blood collection}

Venous blood was collected from a peripheral arm vein at four time points per supplement condition: before supplementation (baseline); after supplementation period and before exercise (PRE); immediately post-exercise (POST); $2 \mathrm{~h}$ post-exercise $(2 \mathrm{H})$. With the exception of POST, all blood samples were

Table 2. Physiological stress in sujects ( $n$ 60) who completed $49 \pm 6$ min of cycling in a hot $\left(38 \pm 2^{\circ} \mathrm{C}\right.$ ), humid $(45 \pm 2 \%)$ environment* $^{*}$

(Mean values with their standard errors)

\begin{tabular}{|c|c|c|c|c|c|c|c|c|c|c|}
\hline \multirow{3}{*}{ Variables } & \multicolumn{10}{|c|}{ Exercise time (min) } \\
\hline & \multicolumn{2}{|c|}{10} & \multicolumn{2}{|c|}{20} & \multicolumn{2}{|c|}{30} & \multicolumn{2}{|c|}{40} & \multicolumn{2}{|c|}{50} \\
\hline & Mean & SE & Mean & SE & Mean & SE & Mean & SE & Mean & SE \\
\hline \multicolumn{11}{|c|}{ Heart rate (bpm) } \\
\hline$B G$ & 147 & 21 & 164 & 17 & 174 & 16 & 176 & 15 & 183 & 11 \\
\hline $\mathrm{PL}$ & 146 & 20 & 168 & 25 & 174 & 16 & 176 & 15 & 182 & 14 \\
\hline \multicolumn{11}{|l|}{ RPE } \\
\hline$B G$ & 11 & 2 & 13 & 2 & 15 & 2 & 16 & 2 & 17 & 2 \\
\hline PL & 12 & 2 & 13 & 1 & 15 & 2 & 16 & 2 & 17 & 1 \\
\hline \multicolumn{11}{|l|}{$T_{\mathrm{C}}\left({ }^{\circ} \mathrm{C}\right)$} \\
\hline$B G$ & $37 \cdot 2$ & 0.5 & $37 \cdot 6$ & 0.5 & 38.2 & 0.5 & 38.6 & 0.5 & 38.9 & 0.5 \\
\hline $\mathrm{PL}$ & $37 \cdot 2$ & 0.4 & 37.5 & 0.5 & 38.1 & 0.5 & 38.6 & 0.5 & 38.9 & 0.4 \\
\hline
\end{tabular}

bpm, Beats per min; BG, $\beta$-glucan; PL, placebo; RPE, rating of perceived exertion; $T_{\mathrm{C}}$, core body temperature.

* Subjects supplemented with either BG or PL (rice flour) for $10 \mathrm{~d}$ before each exercise trial. Heart rate, RPE and $T_{\mathrm{C}}$ were measured to track physiological stress in response to exercise. No significant differences between the conditions were noted. These data demonstrate that the quantity of physiological stress was similar between the conditions. 
collected following 15 min of seated rest. Blood was treated with sodium heparin or EDTA in evacuated tubes to prevent clotting (Becton Dickinson). EDTA plasma was separated and frozen at $-80^{\circ} \mathrm{C}$ for cytokine analysis, while the remaining blood was held at room temperature on an oscillating rocker until used for LPS stimulations or flow cytometry. Samples were processed within $2 \mathrm{~h}$ of collection.

\section{Peripheral blood mononuclear cell isolation}

Peripheral blood mononuclear cells (PBMC) were isolated from whole blood using a standard density gradient method involving Histopaque 1077 (Sigma-Aldrich). EDTA-treated whole blood was diluted 1:2 with PBS and $8 \mathrm{ml}$ of diluted blood were layered onto $4 \mathrm{ml}$ Histopaque in a $15 \mathrm{ml}$ conical centrifuge tube. A total of three tubes ( $24 \mathrm{ml}$ of diluted blood) were separated for each blood sample. After centrifugation $(30 \mathrm{~min}$ at $1000 \mathrm{~g}$ ), the floating PBMC layer was removed from the three separation tubes and combined into a single $50 \mathrm{ml}$ conical centrifuge tube. PBS $(45 \mathrm{ml})$ was added to the pooled PBMC. After two additional washes, PBMC were suspended at $4 \times 10^{6}$ cells $/ \mathrm{ml}$ based on an automated viability count (EMD Millipore ViaCount). PBMC viability was $>97 \%$ for all isolations.

\section{Monocyte phenotyping and concentration}

All flow cytometry supplies were purchased from eBioscience unless otherwise noted. Aliquots of isolated PBMC (300000 cells) were added to $1.2 \mathrm{ml}$ library tubes and incubated with pretitred antibodies against CD14-PerCP-Cy5.5 and CD16-APC. After a 30 min incubation at room temperature in the dark, samples were washed with PBS and re-suspended in a final volume of $300 \mu \mathrm{l}$ ( $0.5 \%$ paraformaldehyde). Samples were then transferred to a round bottom polypropylene ninety-six-well plate for acquisition on a Millipore-Guava easyCyte 6HT-2L (EMD Millipore). A minimum of 35000 monocyte events were acquired for each sample. Monocyte total and subset concentrations were determined by the flow cytometer, which uses a precise volumetric syringe to deliver the sample. Before each set of sample analysis, the easyCyte flow cytometer was checked for calibration and daily variation using standard-sized polystyrene beads (easyCheck; EMD Millipore). Daily variation of fluorescence was $<3 \%$ across all four photomultiplier tubes. Sample flow cytometry standard (FCS) files were acquired uncompensated and analysed offline using FCS Express (version 3.0; De Novo Software). Monocytes were identified based on positive CD14 staining; monocyte subsets were identified based on the differential expression of CD16. Isotype and cells-only tubes were included as negative controls. Classic monocytes were identified as $\mathrm{CD} 14^{+} / \mathrm{CD} 16^{-}$, while pro-inflammatory monocytes were $\mathrm{CD} 14^{+} / \mathrm{CD} 16^{+}$.

\section{Lipopolysaccharide-stimulated cytokine production}

Consistent with methods that we have used previously ${ }^{(21,22)}$, we diluted heparinised whole blood 1:10 with PBS and cultured with LPS $(15 \mu \mathrm{g} / \mathrm{ml})$ overnight $(24 \mathrm{~h})$. LPS was used as a stimulant in the present study because it has the capacity to act on a variety of leucocytes, but primarily monocytes, because these
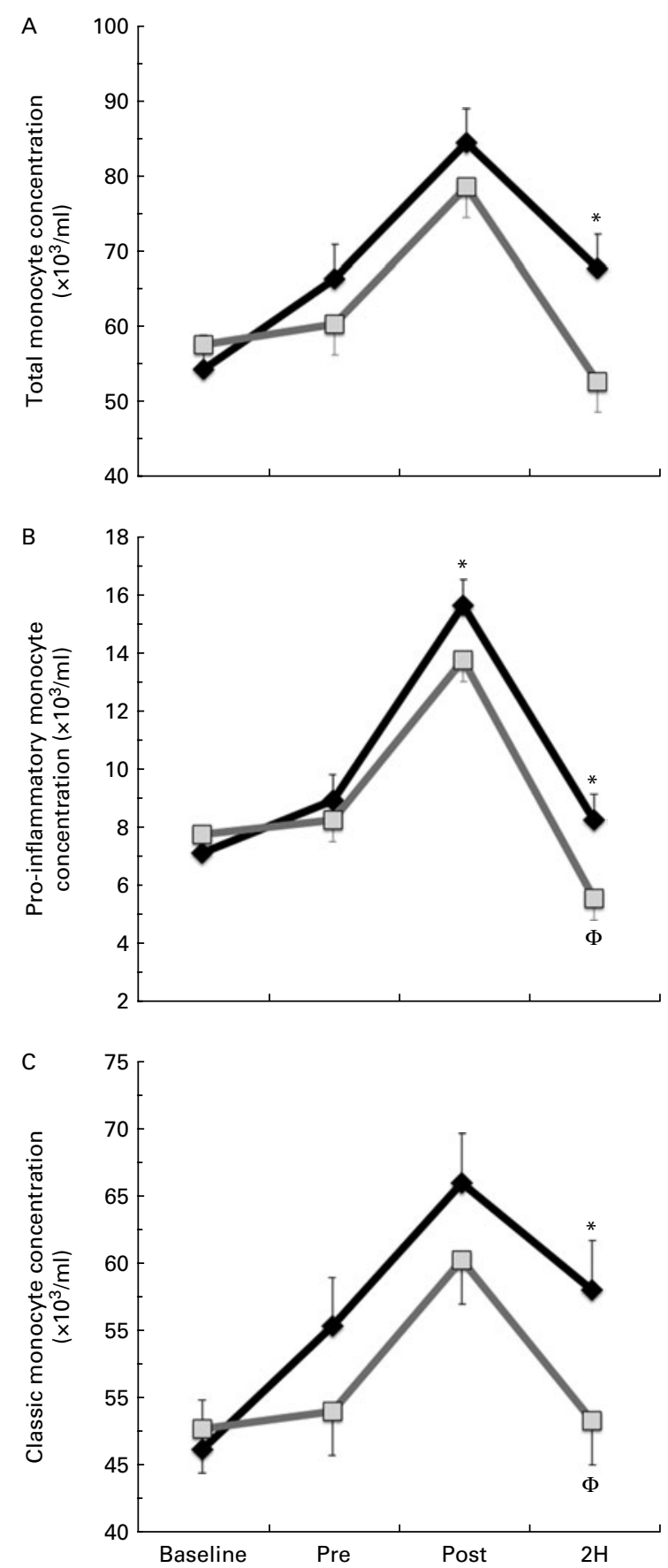

Fig. 2. (a) Total $\left(\mathrm{CD} 14^{+}\right)$, (b) pro-inflammatory $\left(\mathrm{CD} 14^{+} / 16^{+}\right)$and (c) classic $\left(\mathrm{CD} 14^{+} / 16^{-}\right)$monocyte concentration. Venous blood samples were collected before (time point before supplementation (baseline) and after supplementation and before exercise time point (PRE)) and after exercise (immediately post-exercise time point (POST) and $2 \mathrm{~h}$ post-exercise time point $(2 \mathrm{H})$ ) in subjects ( $n$ 60) who completed two trial conditions: baker's yeast $\beta$-glucan ( $250 \mathrm{mg} / \mathrm{d}$ ) and placebo (PL;- - -, rice flour) in a randomised order. Subjects consumed their supplement for $10 \mathrm{~d}$ before the experimental exercise trial in a hot, humid environment (baseline sample collected before the supplementation period). Monocyte concentrations were determined using flow cytometry (EMD Millipore easyCyte 6HT-2L). Significant interaction effects were found for both total $(P=0.026)$ and pro-inflammatory monocyte $(P=0.033)$ concentrations. Classic monocyte concentration showed a similar trend towards an interaction effect as observed in the other two fractions $(P=0 \cdot 117)$. Values are means, with standard errors represented by vertical bars. * Mean value was more significantly different from that of $\mathrm{PL}$ at the same time point. † Mean value was less significantly different from that at PRE for the same condition. 
are the only cells which express the LPS receptor $(C D 14)^{(5)}$. After stimulation, cell-free supernatant was removed and frozen at $-80^{\circ} \mathrm{C}$. Supernatants were thawed and measured for the concentration of twelve cytokines (IL-1 $\beta$, IL-2, IL-4, IL-5, IL-6, IL-7, IL-8, IL-10, IL-12 (p70), IL-13, granulocyte, monocyte colonystimulating factors (GM-CSF) and TNF- $\alpha$ ) using a commercially available flow cytometry-based multiplex bead array (FlowCytomix; eBioscience). The inter- and intra-assay $\mathrm{CV}$ reported by the manufacturer was $<8 \%$.

\section{Plasma cytokine analysis}

EDTA plasma was thawed and analysed using a high-sensitivity, multiplex assay for cytokines (Millipore Milliplex). The test protocol included the measurement of IL-1 $\beta$, IL-2, IL-4, IL-5, IL-6, IL-7, IL-8, IL-10, IL-12 (p70), IL-13, GM-CSF, IFN- $\gamma$ and TNF- $\alpha$. After following the sample processing procedure, labelled magnetic microspheres were acquired on a Luminex MagPix instrument. The low-end detection limit of the assay that we used was $0 \cdot 13 \mathrm{pg} / \mathrm{ml}$ with an inter- and intra-assay CV of $<7 \%$. Manufacturer-provided controls were utilised to track variability in the analysis and all samples were measured on the same day to minimise variability.

\section{Statistical analysis}

All statistical analyses were completed using SPSS (version 19; SPSS, Inc.). Before formal statistical testing, data were analysed for normality and constant error variance. Non-normal data were transformed to stabilise assumptions (noted in the Results section by variable). Exercise response variables were analysed using a two-condition (supplement) $\times$ five-time (exercise) ANOVA with repeated measures on both factors. Immune response variables were analysed using a two (supplement) $\times$ four (blood collection time) ANOVA with repeated measures on both factors. Significance was set at $P<0.05$ and significant $P$ values were adjusted using the Huynh-Feldt method to account for the repeated-measures design. Location of significant effects was determined using a Tukey post hoc test. Data are reported as means with their standard errors.

\section{Results}

\section{Exercise-induced physiological stress}

Subjects completed an average of $49 \pm 6 \mathrm{~min}$ of exercise and the majority were stopped because their $T_{\mathrm{C}}$ reached our safe cut-off of $>39 \cdot 2{ }^{\circ} \mathrm{C}$. Based on the outcome measures that we selected, there was a significant main effect for time for $T_{\mathrm{C}}$ $(P<0.001)$, HR $(P<0.001)$ and rating of perceived exertion $(P<0 \cdot 001)$; however, there was no difference between the conditions (Table 2). The HR response observed in the present study was consistent with an exercise intensity between 60 and $70 \%$ of $\mathrm{VO}_{2 \text { peak. }}$. Based on this analysis, we are confident that the stress associated with the two exercise sessions was not significantly different.
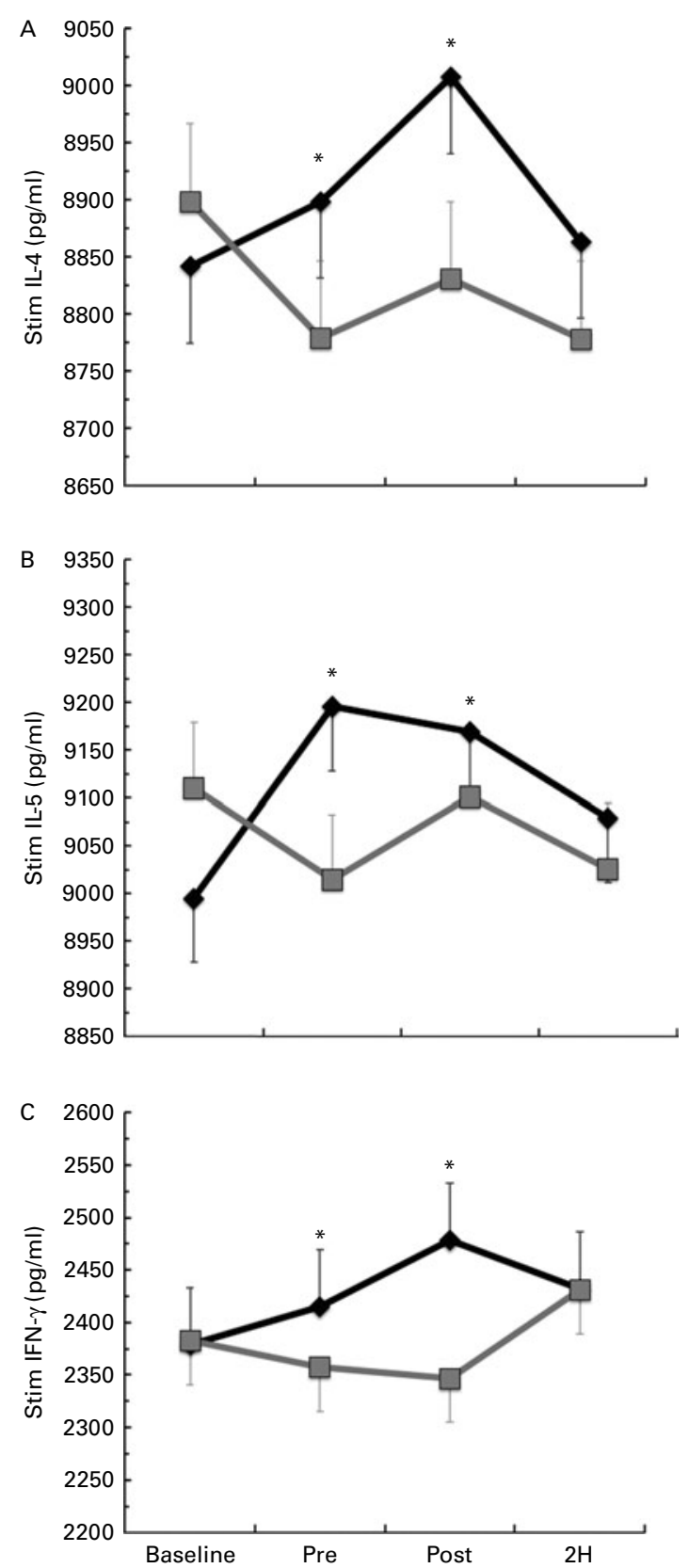

Fig. 3. Lipopolysaccharide (LPS)-stimulated production of (a) IL-4, (b) IL-5 and (c) interferon- $\gamma($ IFN- $\gamma$ ). Venous blood samples were collected before (time point before supplementation (baseline) and after supplementation and before exercise time point (PRE)) and after exercise (immediately post-exercise time point (POST) and $2 \mathrm{~h}$ post-exercise time point $(2 \mathrm{H})$ ) in subjects ( $n$ 60) who completed two trial conditions: baker's yeast $\beta$-glucan (BG,- - -, $250 \mathrm{mg} / \mathrm{d}$ ) and placebo (PL, $\square$-, rice flour) in a randomised order. Subjects consumed their supplement for $10 \mathrm{~d}$ before the experimental exercise in a hot, humid environment (baseline sample collected before the supplementation period). Whole-blood cultures were stimulated for $24 \mathrm{~h}$ with LPS. Cellfree supernatants were analysed for cytokine production using a flow cytometry-based multiplex assay. Significant interaction effects were found for BG over PL at specific time points in IL-4 $(P=0.025)$, IL-5 $(P=0.027)$ and IFN- $\gamma$ $(P=0.011)$. It is important to note that changes in stimulated cytokine production preceded the change in plasma cytokines (Fig. 4). Values are means, with standard errors represented by vertical bars. * Mean value was more significantly different from that of $\mathrm{PL}(P<0.05)$. 


\section{Total and subset monocyte concentrations}

We found a significant condition $\times$ exercise time effect for total monocyte (CD14 ${ }^{+}, P=0.026$; Fig. 2(a)) and pro-inflammatory monocyte $\left(\mathrm{CD} 14^{+} / \mathrm{CD} 6^{+}, P=0.033\right.$; Fig. 2(b)) concentrations. Total monocyte concentration was significantly greater in $\mathrm{BG}$ than in PL at $2 \mathrm{H}$. Pro-inflammatory monocyte concentration was significantly greater in BG than in PL at both POST and $2 \mathrm{H}$. In the $\mathrm{PL}$ condition, $2 \mathrm{H}$ concentration was significantly less than at PRE for both total and pro-inflammatory monocytes $(P<0 \cdot 05)$. While classic monocyte $\left(\mathrm{CD} 14^{+} / \mathrm{CD} 16^{-}\right)$concentration demonstrated a similar pattern, there was no statistically significant interaction effect $(P=0 \cdot 117$; Fig. $2(c))$. The presented concentrations are based on counts taken from PBMC, which are lower than counts determined from the whole blood.

\section{Lipopolysaccharide-stimulated cytokines}

Significant condition $\times$ exercise time interactions were found for the LPS-stimulated cytokines IL-4 ( $P=0.025$; Fig. 3(a)), IL-5 $(P=0.027$; Fig. 3(b)) and IFN- $\gamma(P=0.011$; Fig. 3(c)). For each of these significant cytokines, the location of significance was that the BG condition had significantly greater cytokine production at PRE and POST than PL. It is important to note that changes in stimulated cytokine production preceded the change in plasma cytokines (Fig. 4). All of the apparent BG effects on LPS-stimulated cytokine production occurred before exercise, except for IL-4, which was also significantly increased at $2 \mathrm{H}$ for BG. A significant interaction effect was also found for LPS-stimulated IL-2 $(P=0 \cdot 018)$; however, this difference occurred at baseline and did not demonstrate any other significant differences between BG and PL (data not shown). No significant differences were found between BG and PL for the LPS-stimulated production of IL-1 $\beta$, IL-6, IL-7, IL-8, IL-10, IL-12 (p70), IL-13, GM-CSF or TNF- $\alpha$.

\section{Plasma cytokines}

Significant condition $\times$ exercise time effects were found for plasma concentrations of IL-4 ( $P=0.042 ; \quad$ Fig. 4(a)), IL-7 $(P=0.047$; Fig. 5(a)), IL-8 ( $P=0 \cdot 049$; Fig. 5(b)), IL-10 ( $P=0.033$; Fig. 5(c)) and IFN- $\gamma(P=0.047$; Fig. 4(c)). There was also a trend towards significance for IL- $5(P=0.053$; Fig. $4(\mathrm{~b}))$. For each of these cytokines, the location of significance was the same; the BG condition was significantly greater than PL at $2 \mathrm{H}$. Also, for IL- 4 and IL-5, BG was greater than PL at PRE. We found a significant main effect for time (no supplement effect) for IL-1 $\beta$ $(P=0.017)$, IL-6 $(P<0.0001)$, TNF- $\alpha P<0.0001)$ and GM-CSF $(P=0.06)$ (Table 3 ). The changes observed in both conditions are consistent with a typical post-exercise pro-inflammatory response (IL-1 $\beta$, IL-6, TNF- $\alpha$ and GM-CSF). No significant main or interaction effects were found for IL-2, IL-12 (p70) or IL-13.

\section{Discussion}

The key findings of the present study were that supplementation for $10 \mathrm{~d}$ with $250 \mathrm{mg}$ of baker's yeast BG per d before a bout of cycling in the heat increased total and pro-inflamma-
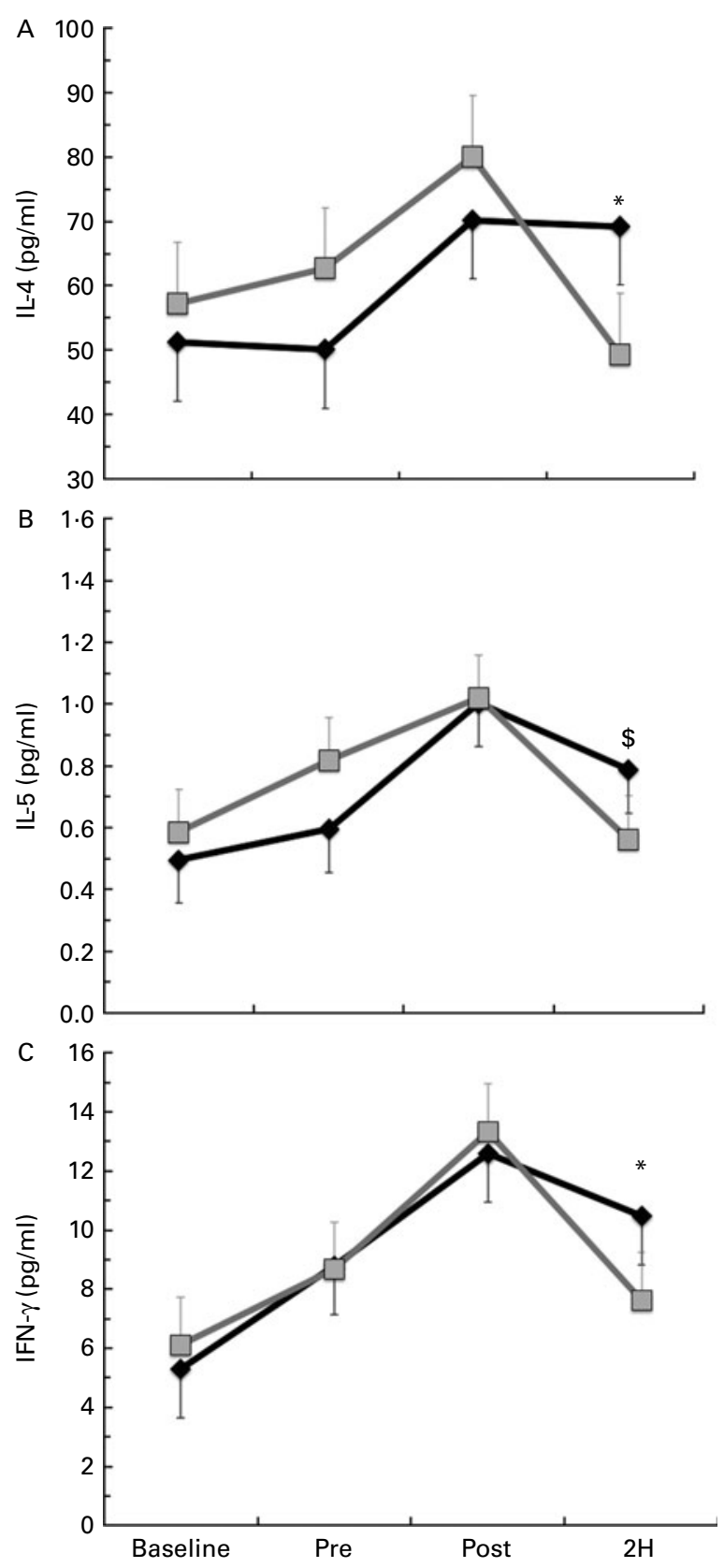

Fig. 4. Plasma concentration of (a) IL-4, (b) IL-5 and (c) interferon- $\gamma$ $($ IFN- $\gamma$ ). Venous blood samples were collected before (time point before supplementation (baseline) and after supplementation and before exercise time point (PRE)) and after exercise (immediately post-exercise time point (POST) and $2 \mathrm{~h}$ post-exercise time point $(2 \mathrm{H})$ ) in subjects $(n 60)$ who completed two trial conditions: baker's yeast $\beta$-glucan $(B G,-\mathbf{-}, 250 \mathrm{mg} / \mathrm{d}$ ) and placebo ( $\mathrm{PL},-\square$, rice flour) in a randomised order. Subjects consumed their supplement for $10 \mathrm{~d}$ before the experimental exercise in a hot, humid environment (baseline sample collected before the supplementation period). Plasma was analysed using a high-sensitivity multiplex cytokine kit (EMD Millipore Milliplex kit and Luminex MagPix instrument). Significant interaction effects were found for $B G$ over $P L$ at specific time points in IL-4 $(P=0.042)$ and IFN- $\gamma(P=0.047)$. There was a trend towards significance for this effect in IL-5 $(P=0.053)$. It is important to note that changes in plasma cytokines followed changes in stimulated cytokine production (Fig. 3). Values are means, with standard errors represented by vertical bars. *Mean value was more significantly different from that of $P L$ $(P<0.05)$. † Mean value was more significantly different from that of $\mathrm{PL}$ $(P$ for trend $=0.053)$. 

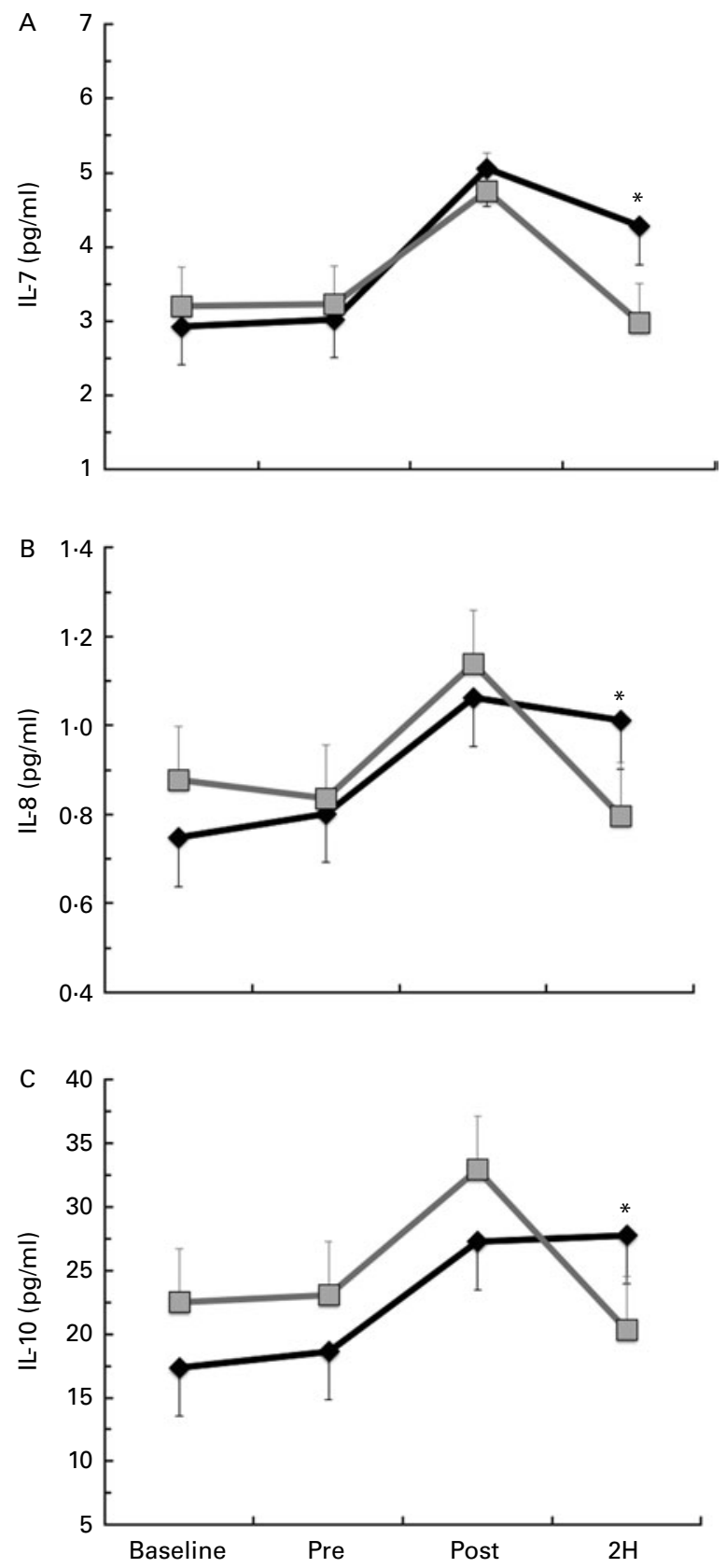

Fig. 5. Plasma concentration of (a) IL-7, (b) IL-8 and (c) IL-10. Venous blood samples were collected before (time point before supplementation (baseline) and after supplementation and before exercise time point (PRE)) and after exercise (immediately post-exercise time point (POST) and $2 \mathrm{~h}$ post-exercise time point $(2 \mathrm{H})$ ) in subjects $(n 60)$ who completed two trial conditions: baker's yeast $\beta$-glucan $(B G,-, 250 \mathrm{mg} / \mathrm{d})$ and placebo $(\mathrm{PL},-\mathrm{-}-$, rice flour) in a randomised order. Subjects consumed their supplement for $10 \mathrm{~d}$ before the experimental exercise in a hot, humid environment (baseline sample collected before the supplementation period). Plasma was analysed using a high-sensitivity multiplex cytokine kit (EMD Millipore Milliplex kit and Luminex MagPix instrument). Values are means, with their standard errors represented by vertical bars. * Mean value was more significantly different from that of $\mathrm{PL}(P<0.05)$.

tory monocyte concentrations after exercise, increased LPSstimulated cytokine production before exercise and increased plasma cytokine concentrations after exercise. While other published studies have examined BG supplementation, the results have varied due to the structural differences in BG from different sources ${ }^{(23-26)}$. To our knowledge, this is the first published report demonstrating that supplementation with a well-characterised form of baker's yeast $\mathrm{BG}^{(15)}$ alters the post-exercise innate immune system response.

For PL, we observed a $17 \%$ decline in both total and proinflammatory monocyte concentrations between PRE and $2 \mathrm{H}$, compared with only a $3 \%$ decline with $\mathrm{BG}$. The $2 \mathrm{H}$ decline observed in the PL condition was consistent with previous reports from our laboratory and others of exerciseinduced immunosuppression ${ }^{(21,27,28)}$. It appears that BG supplementation maintains the blood monocyte circulation, and counters the monocytopenia that we observed in the PL condition post-exercise. A BG-induced increase in monocytes at $2 \mathrm{H}$ may have important implications for the global immune response ${ }^{(29)}$. Monocytes play a role in tissue-specific Th1 and Th2 responses and are know to be activated by Th1 and Th2 cytokines $^{(2,7,30)}$. Iijima et al $^{(31)}$ reported that an increase in circulating pro-inflammatory monocyte concentration resulted in improved Th1 response in the peripheral tissue. The response that we observed at $2 \mathrm{H}$ with $\mathrm{BG}$ was very similar to the findings of Iijima et al. For PL, we observed the effects that were completely opposite of BG and consistent with the typical exercise response ${ }^{(32)}$.

In addition to changes in circulating monocyte concentration, we also measured changes in both plasma cytokine levels and LPS-stimulated cytokine production by peripheral blood cells. Some of the targeted cytokines demonstrated changes in both measures, while others only demonstrated changes in plasma after BG supplementation. We found that BG increased LPSstimulated production of IFN- $\boldsymbol{\gamma}$, IL- 4 and IL- 5 before exercise and increased in plasma IFN- $\gamma$, IL- 4 and IL- 5 (trend towards significance) concentrations at $2 \mathrm{H}$. Further interpretation of this finding suggests that BG may potentiate the ability of monocytes to increase their cytokine output after exercise, resulting in greater plasma cytokine concentrations. This increased capacity to respond to the LPS challenge is similar to the enhanced response of innate immune cells after exposure to yeast BG reported in animal model systems. Yeast BG has been shown to bind to complement receptor 3 on neutrophils and other innate immune cells ${ }^{(33)}$. With BG fragments bound to complement receptor 3 , the 'primed' neutrophils are more responsive to chemotactic stimuli ${ }^{(13,34)}$ as well as having enhanced levels of phagocytosis $^{(14)}$ and oxidative burst ${ }^{(10,12,33)}$.

Since LPS stimulation mimics a bacterial challenge of the immune system ${ }^{(35)}$, the increased cytokine production of LPSstimulated peripheral blood cells after BG supplementation may be indicative of increased monocyte functional capacity at rest. The cytokine response to LPS stimulation observed with PL was the opposite of BG, but consistent with what others have reported in response to exercise-induced stress ${ }^{(32)}$. Since cytokines are regulators of many immune system processes, based on the data presented here, we speculate that BG supplementation improves monocyte function, which may have downstream effects on other aspects of the innate immune response.

We also observed changes in plasma cytokines that are associated with lymphocyte proliferation (IL-7) and chemotaxis (IL-8), and that exert anti-inflammatory actions 
Table 3. Plasma cytolcines in subjects $(n 60)$ who completed $49 \pm 6$ min of cycling in a hot $\left(38 \pm 2^{\circ} \mathrm{C}\right.$ ), humid $(45 \pm 2 \%)$ environment $\dagger$ (Mean values with their standard errors)

\begin{tabular}{|c|c|c|c|c|c|c|c|c|}
\hline \multirow[b]{2}{*}{ Variables $(\mathrm{pg} / \mathrm{ml})$} & \multicolumn{2}{|c|}{ Baseline } & \multicolumn{2}{|c|}{ PRE } & \multicolumn{2}{|c|}{ POST } & \multicolumn{2}{|c|}{$2 \mathrm{H}$} \\
\hline & Mean & SE & Mean & SE & Mean & SE & Mean & SE \\
\hline \multicolumn{9}{|l|}{ IL-1 $1 \beta$} \\
\hline$B G$ & 0.51 & 0.13 & 0.68 & 0.15 & 0.88 & 0.16 & 0.81 & 0.16 \\
\hline PL & 0.57 & 0.08 & 0.81 & 0.13 & 1.05 & 0.19 & 0.69 & 0.13 \\
\hline \multicolumn{9}{|l|}{ IL-6 } \\
\hline$B G$ & 1.53 & 0.26 & 1.51 & 0.24 & 2.42 & 0.37 & 1.80 & 0.29 \\
\hline PL & 1.64 & 0.26 & 1.84 & 0.38 & $2 \cdot 66$ & 0.40 & 1.64 & 0.28 \\
\hline \multicolumn{9}{|l|}{ TNF- $\alpha$} \\
\hline BG & 1.51 & 0.20 & 1.82 & 0.33 & $3 \cdot 16^{\star}$ & 0.45 & 2.22 & 0.39 \\
\hline $\begin{array}{c}\mathrm{PL} \\
\mathrm{GM}-\mathrm{CSF}\end{array}$ & 1.73 & 0.22 & $1 \cdot 84$ & 0.26 & $3.09^{\star}$ & 0.45 & $1 \cdot 64$ & 0.25 \\
\hline$B G$ & $8 \cdot 15$ & $1 \cdot 31$ & 13.91 & $4 \cdot 10$ & $18 \cdot 57^{\star}$ & 3.65 & 11.01 & 1.77 \\
\hline PL & $9 \cdot 81$ & 1.76 & $12 \cdot 61$ & $2 \cdot 31$ & $20.57^{\star}$ & $4 \cdot 18$ & 11.08 & $2 \cdot 51$ \\
\hline
\end{tabular}

Baseline, time point before supplementation; $\mathrm{PRE}$, after supplementation and before exercise time point; $\mathrm{POST}$, immediately post-exercise time point; $2 \mathrm{H}, 2 \mathrm{~h}$ post-exercise time point; BG, $\beta$-glucan; PL, placebo; GM-CSF, granulocyte, monocyte colony-stimulating factors.

${ }^{*}$ Mean value was significantly different from those at the other time points $(P<0.05)$

† Subjects supplemented with either BG or PL (rice flour) for $10 \mathrm{~d}$ before each exercise trial. Venous blood samples were collected before supplementation (baseline), PRE, POST and 2H. Plasma IL-1 $\beta$, IL-6, TNF- $\alpha$ and GM-CSF were measured as part of a Milliplex High-Sensitivity cytokine kit. Other results are presented in Figs. 4 and 5.

$\left(\right.$ IL-10) ${ }^{(29)}$; however, these cytokines did not have a corresponding change in LPS-stimulated response, suggesting that monocytes may not be the sole plasma cytokine source following exercise with BG supplementation.

This finding is plausible for IL-7 and IL-8, which are not produced by monocytes; however, it fails to explain the source of IL-10, since it is produced by monocytes. IL-7 has been demonstrated to improve $\mathrm{T}$-cell and $\mathrm{B}$-cell proliferation in response to antigenic challenge and IL- 8 is an inducer of chemotaxis in neutrophils ${ }^{(29)}$. In the present study, BG supplementation increased IL- 7 and IL- 8 at $2 \mathrm{H}$, which, based on their respective function, may be associated with improved T-cell and B-cell proliferative capacity and an increased recruitment of neutrophils to the tissue compartment. IL-10 is a potent anti-inflammatory cytokine, which we found to increase at $2 \mathrm{H}$ in $\mathrm{BG}$ compared with PL. Since we found a similar pro-inflammatory response to exercise in both supplement groups (elevated IL-1 $\beta$, IL-6, TNF- $\alpha$ and GM-CSF), it is plausible that BG supplementation provided additional anti-inflammatory protection that was not afforded in the PL condition. More research is needed to understand the immunological source of IL-7, IL-8 and IL-10 in response to BG supplementation.

The changes that we observed in the present study demonstrate how BG supplementation before exercise alters the post-exercise innate immune response. Some of the changes that we observed with BG supplementation were small; however, it is important to note that the study was appropriately powered to detect these changes, which did reach statistical significance. Collectively, the observed changes may have implications for post-exercise immune surveillance and subsequent infection risk.

In summary, in the present study, we found that $10 \mathrm{~d}$ of supplementation with a defined source of baker's yeast BG increased total and pro-inflammatory monocyte concentrations after exercise, increased LPS-stimulated cytokines
(IL-2, IL-4, IL-5 and IFN- $\gamma$ ) before exercise and plasma cytokine (IL-4, IL-5, IL-7, IL-8, IL-10 and IFN- $\gamma$ ) concentrations after exercise. We also observed a post-exercise pro-inflammatory response (IL-1 $\beta$, IL-6, TNF- $\alpha$ and GM-CSF) regardless of supplement that was consistent with previous reports from our laboratory and others. These findings support the notion that supplementation with the Wellmune WGP form of baker's yeast $B G$ before exercise altered the typical post-exercise innate immune response. More research is needed to understand what the clinical implications of the observed changes are.

\section{Acknowledgements}

We would like to thank Kelley Strohacker, Tanya Halliday, Stephanie Collins, Danielle Eagan and Nicole Impero for their various contributions to this study. We would also like to thank all our subjects who participated in the present study, without their commitment the present study would not have been possible. Funding for the present study was provided by Biothera, the Immune Health Company. The authors were not directly paid for the completion of this study and declare that there is no conflict of interest. K. C. C., W. L. B. and B. K. M. participated in the data collection, analysis and manuscript preparation. T. D. and A. A. contributed to the data collection and analysis. B. K. M. designed and directed the overall study.

\section{References}

1. Woods JA, Davis JM, Smith JA, et al. (1999) Exercise and cellular innate immune function. Med Sci Sports Exerc 31, 57-66.

2. McFarlin BK, Flynn MG, Stewart LK, et al. (2004) Carbohydrate intake during endurance exercise increases natural killer cell responsiveness to IL-2. J Appl Physiol 96, 271-275.

3. Walsh NP \& Whitham M (2006) Exercising in environmental extremes: a greater threat to immune function? Sports Med 36, 941-976. 
4. Pedersen BK \& Ullum H (1994) NK cell response to physical activity: possible mechanisms of action. Med Sci Sports Exerc 26, 140-146.

5. Gleeson M, McFarlin B \& Flynn M (2006) Exercise and Tolllike receptors. Exerc Immun Rev 12, 34-53.

6. McFarlin BK, Hutchison AT \& Kueht ML (2008) Knowledge of carbohydrate consumption does not alter natural killer cell activity following an acute bout of high-intensity aerobic exercise. Appl Physiol Nutr Metab 33, 1007-1012.

7. Steensberg A, Toft AD, Bruunsgaard H, et al. (2001) Strenuous exercise decreases the percentage of type $1 \mathrm{~T}$ cells in the circulation. J Appl Physiol 91, 1708-1712.

8. McFarlin BK, Flynn MG \& Hampton T (2007) Carbohydrate consumption during cycling increases in vitro NK cell responses to IL-2 and IFN-gamma. Brain Behav Immun 21, 202-208.

9. McFarlin BK, Flynn MG, Phillips MD, et al. (2005) Chronic resistance exercise training improves natural killer cell activity in older women. J Gerontol A Biol Sci Med Sci 60, 1315-1318.

10. Allendorf DJ, Yan J, Ross GD, et al. (2005) C5a-mediated leukotriene B4-amplified neutrophil chemotaxis is essential in tumor immunotherapy facilitated by anti-tumor monoclonal antibody and beta-glucan. J Immunol 174, 7050-7056.

11. Driscoll M, Hansen R, Ding C, et al. (2009) Therapeutic potential of various beta-glucan sources in conjunction with anti-tumor monoclonal antibody in cancer therapy. Cancer Biol Ther 8, 218-225.

12. Lavigne LM, Albina JE \& Reichner JS (2006) Beta-glucan is a fungal determinant for adhesion-dependent human neutrophil functions. J Immunol 177, 8667-8675.

13. Li B, Allendorf DJ, Hansen R, et al. (2007) Combined yeast \{beta\}-glucan and antitumor monoclonal antibody therapy requires $\mathrm{C} 5 \mathrm{a}$-mediated neutrophil chemotaxis via regulation of decay-accelerating factor CD55. Cancer Res 67, 7421-7430.

14. Wakshull E, Brunke-Reese D, Lindermuth J, et al. (1999) PGG-glucan, a soluble beta-(1,3)-glucan, enhances the oxidative burst response, microbicidal activity, and activates an NF-kappa B-like factor in human PMN: evidence for a glycosphingolipid beta-(1,3)-glucan receptor. Immunopharmacology 41, 89-107.

15. us Pharmacopiea (2011) Beta glucan from bakers yeast (Saccharomyces cerevisiae). In Food Chemicals Codex, 7th ed, 3rd supplement [us Pharmacopiea, editor]. New York: US Pharmacopiea.

16. Talbott ST \& Talbott J (2009) Effect of beta 1,3/1,6 glucan on upper respiratory tract infection symptoms and mood state in marathon athletes. J Sports Sci Med 8, 509-515.

17. Harger-Domitrovich SG, Domitrovich JW \& Ruby BC (2008) Effects of an immunomodulating supplement on upper respiratory tract infection symptoms in wildland firefighters. Med Sci Sports Exerc 40, S353.

18. McFarlin BK, Mitchell JB, McFarlin MA, et al. (2003) Repeated endurance exercise affects leukocyte number but not NK cell activity. Med Sci Sports Exerc 35, 1130-1138.

19. McFarlin BK \& Mitchell JB (2003) Exercise in hot and cold environments: differential effects on leukocyte number and NK cell activity. Aviat Space Environ Med 74, 1231-1236.

20. Ehrman JK (2010) American College of Sports Medicine. ACSM's Resource Manual for Guidelines for Exercise Testing and Prescription, 6th ed. Philadelphia, PA: Wolters Kluwer Health/Lippincott Williams \& Wilkins.

21. McFarlin BK, Flynn MG, Campbell WW, et al. (2004) TLR4 is lower in resistance-trained older women and related to inflammatory cytokines. Med Sci Sports Exerc 36, 1876-1883.

22. McFarlin BK, Flynn MG, Campbell WW, et al. (2006) Physical activity status, but not age, influences inflammatory biomarkers and toll-like receptor 4. J Gerontol A Biol Sci Med Sci 61, 388-393.

23. Bergendiova K, Tibenska E \& Majtan J (2011) Pleuran (betaglucan from Pleurotus ostreatus) supplementation, cellular immune response and respiratory tract infections in athletes. Eur J Appl Physiol 111, 2033-2040.

24. Murphy EA, Davis JM, Carmichael MD, et al. (2009) Benefits of oat beta-glucan and sucrose feedings on infection and macrophage antiviral resistance following exercise stress. Am J Physiol Regul Integr Comp Physiol 297, R1188-R1194.

25. Nieman DC, Henson DA, McMahon M, et al. (2008) Beta-glucan, immune function, and upper respiratory tract infections in athletes. Med Sci Sports Exerc 40, 1463-1471.

26. Murphy EA, Davis JM, Brown AS, et al. (2008) Benefits of oat beta-glucan on respiratory infection following exercise stress: role of lung macrophages. Am J Physiol Regul Integr Compar Physiol 294, R1593-R1599.

27. Simpson RJ, McFarlin BK, McSporran C, et al. (2009) Toll-like receptor expression on classic and pro-inflammatory blood monocytes after acute exercise in humans. Brain Behav Immun 23, 232-239.

28. Flynn MG, McFarlin BK, Phillips MD, et al. (2003) Toll-like receptor 4 and CD14 mRNA expression are lower in resistive exercise-trained elderly women. J Appl Physiol 95, $1833-1842$.

29. Walsh NP, Gleeson M, Shephard RJ, et al. (2011) Position statement. Part one: immune function and exercise. Exerc Immunol Rev 17, 6-63.

30. Ibfelt T, Petersen EW, Bruunsgaard H, et al. (2002) Exerciseinduced change in type 1 cytokine-producing CD $8+\mathrm{T}$ cells is related to a decrease in memory T cells. J Appl Physiol $\mathbf{9 3}$, 645-648.

31. Iijima N, Mattei LM \& Iwasaki A (2011) Recruited inflammatory monocytes stimulate antiviral Th1 immunity in infected tissue. Proc Natl Acad Sci U S A 108, 284-289.

32. Lancaster GI, Halson SL, Khan Q, et al. (2004) Effects of acute exhaustive exercise and chronic exercise training on type 1 and type $2 \mathrm{~T}$ lymphocytes. Exerc Immunol Rev 10, 91-106.

33. Hong F, Yan J, Baran JT, et al. (2004) Mechanism by which orally administered beta-1,3-glucans enhance the tumoricidal activity of antitumor monoclonal antibodies in murine tumor models. J Immunol 173, 797-806.

34. Tsikitis VL, Morin NA, Harrington EO, et al. (2004) The lectin-like domain of complement receptor 3 protects endothelial barrier function from activated neutrophils. J Immunol 173, 1284-1291.

35. Wang PL, Oido-Mori M, Fujii T, et al. (2001) Heterogeneous expression of Toll-like receptor 4 and downregulation of Toll-like receptor 4 expression on human gingival fibroblasts by Porphyromonas gingivalis lipopolysaccharide. Biochem Biophys Res Commun 288, 863-867. 